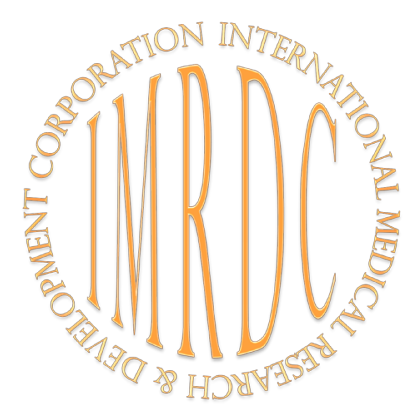

\title{
A Mythologem as a Determinant of Goal-Directed Behavior
}

\author{
Alexander G. Kruglov, PhD, ScD \\ Central Research Institute of Radiation Diagnosis \\ Moscow, the Russian Federation
}

\begin{abstract}
The construction of a principled and generalized model of a mythologem (ML) as psychogenic structure having psychological, electric, magnetic, hemodynamic, and time equivalents is the result of this study. Periodic invariant sensory patterns, creating a dominant center, form a stable neural network, the result of which is an ML. Within the framework of the ML model construction, the partial identity of ML and a motive, the image of the goal (IG) is accepted. The location of the IG was attributed to the expected result, which is located outside the attainable. The ML equivalents were defined as properties of a unified psychogenic structure initiating the forms of goal-directed behavior, which are unable to achieve the final goal, and a homeostatic balance, as a final result. We believe that anticipatory, prospective formation of IG, initiating adaptive forms of behavior, is a manifestation of a general principle of "anticipatory response" (the formation of the future in the present moment) inherent for somatic and mental spheres of human ontogenesis. (Int J Biomed. 2015;5(4):231-234.)
\end{abstract}

Keywords: mythologem; the image of the goal; motive; goal-directed behavior; subjective time.

\section{Abbreviations}

ML, mythologem; IG, the image of the goal; MG, motivational gradient; ST, subjective time; DC, dominant center; GDB, goal-directed behavior; EDS, electrodynamic structure

\section{Introduction}

In accordance with modern ideas set out in the research of a number of authors, ML is generally regarded as a figurative, symbolic way to display reality, which is required when the reality does not fit into a formal-logic and abstract display. ML is a stable construct, generally reflecting reality in the form of sensory-specific associations perceived as objective reality [1]. Man lives in the power of illusion, which is so strong that it seems as normal consciousness [2]. ML is capable of directing the activity of the individual and mobilizing this activity to achieve significant goals [3].

The objective of this study was to build a model of ML as a psychogenic, stable construction having psychological, electric, magnetic, hemodynamic, and timing parameters of the neural networks (associations) that provide the formation, the initiation, and the existence of the ML. Understanding

*Corresponding author: Alexander G. Kruglov, PhD, ScD. Deputy Director of Central Research Institute of Radiation Diagnosis. Moscow, the Russian Federation.E-mail:krag48@mail.ru the concept of the formation and stability of ML will help in finding the antithesis of the confidence [4] in the invincibility of ML.

\section{Materials and Methods}

To create the model of ML as a meaningful unity of the neural networks having the psychological, electrical, magnetic, hemodynamic, and timing parameters, we used our own previously published data (the parameters of hemodynamics and biochemistry of the human brain obtained by invasive methods) [5-8] and data of other authors.

\section{Result and Discussion}

ML fixates the sequence, the order of the representation elements about the reality, and serves as the conceptual basis of behavior that allows us to functionally consider ML as a "motive" for specific forms of behavior. The motive is the cause of behavior and consists of a set of dispositions, the most important of which is the need [9]. A need is defined as a state of dissatisfaction of the organism (the personality) and a 
deficit of what is required to maintain homeostatic balance of the organism (the personality). A special feature of "organized matter" is the selectivity of the reaction that is the subject of urgent needs, i.e. elimination of the gradient between the current status and the target status, the result for which the activity is undertaken, namely, the image of the future results generated by the subject, defined as IG. IG is a leading element of the conscious regulatory process. We think it possible, within ML modeling, to determine the partial identity of the ML and IG (ML IG).

Association of the interneurons activated to provide GDB, whose ultimate aim is the achievement of parametric equalization and the state of homeostatic balance, we consider as a possible substrate for ML as the long-term circular structure [5]. We believe that the periodic afferent stimulation, which has a constant parametric set during the exposure time (the invariant sensory pattern - training, persuasion, suggestion, etc.) is the initial step in the formation of $\mathrm{ML} / \mathrm{IG}$. The sensory afferent stimulation of synapses by the repetitive, monotonous sensory patterns (short- and long-term potentiation) creates the "beaten paths" to facilitate the passage of this particular set of patterns. The formation of the neural "beaten paths" during a quite long afferentation leads to the creation of the sustainable neuronal formation - a dominant center (DC). A DC with the properties of persistence, the concentration of an excitation from other nerve centers, the inhibition of functionally incompatible centers (i.e. filtering the input parameters of the sensory information) determines the nature of the current reactions to the time of its existence. We believe that this consistently structured neural formation creates the IG. It should be noted that the mind has the ability to create the pathological dominants, determinant, and systems [10], and to design images which do not reflect the reality but are accompanied by the sensory, cognitive, and behavioral equivalents with preserved consciousness (phantom pain, some hallucinatory syndromes).

The activity of neurons reflects their involvement in the system, where the organization of all processes is the informative equivalent of the result, i.e. the future [9]. The completion of the IG construction as a result of the successive sensory stimulation, in our opinion, is subjectively experienced as a state known by definition as insight. The IG creation activates the efferent structure described by us [5] as a motivational gradient (MG). The ultimate goal of MG implemented through GDB, is to achieve the status of parametric balance, homeostatic balance. The achievement of the expected utilitarian goals (food, sexual behavior, etc.) leads to the restoration of homeostasis, i.e. elimination of the MG. In contrast to the short-term and "tactical" goals, the forwardlooking plans for the achievement of the "strategic" goals covering large time intervals have the discrete structure of $\mathrm{MG}$ and the subjective time (ST) (i.e. phenomena, accompanied by the expected changes in the parameters as a result of GDB, may be formed in the interval-separated time periods, at the same time being in the plot(story)-caused sequence of the events) [5].

ML, being a set of universalized symbols, has an ambivalent nature, being both a complete construct and an integral part (element) of the new constructs. Two main interacting elements of a symbol, an image and a meaning, exist only inside of the interpretations [11] where the bijective isomorphic coordinate accordance of any operational IG to the final (ideal) IG is impossible. At the same time, there is convincing evidence of a homomorphism, where full compliance with the ideal IG is impossible, but some degree of similarity is achieved. In the absence of the concretized and utilitarian form (image) during our movement to a symbolic goal, new coordinating and interpretive combinations of IG and a semantic content are formed, which create a fractional and operative image without the possibility of reaching the ideal IG. Consequently, the movement towards a symbolic goal (ML) which has not the specifics of the utilitarian goals and time constraints, creates a new degree of similarity and cannot be completed. In other words, the ML construct has the nature of the expected IG located outside of the actual achievable goal.

Considering the vector of GDB as the direction of a speedy change in the scalar variables, whose values are changed from one point in space to another, we define a "zero" (starting) point of ST in the structure of ML on the expected result of the action, i.e. IG. In psychological time, only the expected outcome (goal), having fixed coordinate specifications, can be taken as an abstract point (which is in the future), while the present is always a certain time interval [12]. Thus, the IG formation is a psycho-physiological stimulus and starting point in the formation of the grading scale of the result achievement, namely, $\mathrm{ST}$. Perhaps a prediction exists as a principle (probably genetic) of the formation of an organism (the personality). In 1930, G.E. Coghill [13] described how during embryogenesis the anatomical structures are formed long before their demand in future behavior (anticipatory structure versus the function). Several authors [14] have described the neurobiological basis of the phenomenon of the "mental anticipatory response." IG formation demonstrates the "anticipatory function" versus the structure.

ML/IG formed by the repetitive patterns of sensory impact on the object has the expected time of existence until the achievement of the ultimate goal, the elimination of the imbalance and the restoration of the parametric equilibrium of the homeostasis. The fundamental properties of the living organisms belonging to the open nonequilibrium systems are their vibrational nature. The organism as a unified formation exists due to the synchronization of the rhythmic changes (the adaptive oscillations) at all hierarchical levels, from the molecular-cellular level to the organism level. Since the ultimate goal (IG) is unattainable, it is impossible to eliminate the imbalances and the restore the homeostatic balance, regardless of the amplitude and duration of the adaptive vibrational changes. The existence of the permanent IG/ML inducing the vector activity initiates the "vibrational" search for forms and vectors of GDB, which is implemented by the cognitive and behavioral activity. The strategies of the IG/ML implementation with (slightly) ineffective negative reverse relations and the conservation (increasing) of the reverse positive relations (filtration of the input sensory information), result in a loss of the correction-concordance of the GDB 
stages to the intrasystem and integrative mechanisms of the central nervous system. In addition, the amplitude of the adaptive and search oscillations of homeostasis decreases, a resistance increases, and the system becomes rigid. The natural result is the development of various forms and degrees of change in thinking and consciousness, as well as reduced energy potential and other manifestations of a long homeostatic imbalance and desynchronosis.

The formation of the activity of the neuronal association in the form of the electrodynamic structure (EDS) circulating through the neural network until the achievement of the result is accompanied by the metabolic support of the exchange fields of the activated neurons [8]. The electrical field of the neuron is largely flattened for the external registrars due to nonlinear dissemination of EDS and the electrical insulating properties of the membranes of astrocytes and meninges, which perhaps explains the still existing differences in EEG decoding. The increase in blood flow (i.e. number and speed of blood flows) through the vessels of the brain tissue is an haemodynamic equivalent of this activity. In the tissue in the cortex and white matter of the brain, the regional linear (in the radial vessels) and local (functional hyperemia) streams of the charged particles of blood are intensified. In accordance with V.P. Oleinik [15], the blood is regarded as an electrolyte, while the bloodstream is regarded as a conductor placed in an external magnetic field. In a moving conducting fluid, the ponderomotive forces directed perpendicular to the linear movement of the blood - the magnetohydrodynamic forces (a magnetic dipole) - are formed. Magnetic properties of blood flow (electrolytes), having an essentially linear (radial vessels) character in the regional supply of the increased metabolism of the exchange fields of brain tissue and spreading perpendicularly to the axial potential do not have the insulating barriers in the form of biological tissue, as an interaction with intracerebral structures, as well as for the external recorders. In biological systems, the effect of an induction occurs under impact of the extremely weak (nano- and picotesla) alternating magnetic fields, where the primary receptor is a water-salt component, while the primary target is the nuclear spin of hydrogen atoms [16].

The alternating electric fields, with the parameters imitating the Schumann's resonances (Konig, 1974: cited by Lednev VV et al. [16]) and defining the circadian rhythms (Wever, 1974: cited by Lednev VV et al. [16]), influence the psycho-physical condition of the person. The match between the Schumann resonance frequency and the alpha-rhythm of the human brain $(7.83 \mathrm{~Hz})$ suggests that the response to the external action depends on a matching (resonance) with frequency parameters which are identical (close) to the characteristics of the intracerebral electrical and magnetic dipoles (the neural circuits, including an inactive).

The data of Konig, Wever, et al., in our opinion, reflect the external manifestation of the activity of neuronal structures of the first signal system of the brain activated due to external influences: an induction by external magnetic fields. We believe that the main mechanism of this interaction is a response of the brain (neural) structures with electromagnetic properties (dipoles) to the external stimulus with identical (close) frequency characteristics (resonance). In the absence of the primary afferent sensory information from the known receptors, we consider the possibility of the direct resonance effect on the neural structures of the brain during exposure to the external magnetic field with the actualization of the brain dipoles coinciding in frequency characteristics. A demonstrative example of this effect, possibly related to the unconditioned reflexes, in our opinion, is animals sensing that an earthquake is about to happen and fleeing from it, even at a distance of $200 \mathrm{~km}$ from the epicenter. Thus, by our assumption, the initiator of mental activity, along with the sensor variables, may be an external vector of magnetic induction with the effect of a direct impact (resonance) on the neuronal circuits (magnetic dipoles) of the brain.

We believe that the registration of the magnetic fields arising during the activation of mental activity largely relates to the parameters of the resulting multipole magnetic field that is a result of vector addition of magnetic dipoles: EDS of the activated neural associations and regional vessels (interdependent distributed systems) having different latency intervals and angular vector differences. We also believe that the differences in the genesis of the magnetic induction should be considered during detailed coding of the results of the magnetographic mapping.

ML, in our opinion, may have objective, measurable parameters: 1) voltage variation of the magnetic fields of the brain of different origin; 2) hemodynamic equivalent with a change in blood flow and shape of the vessel in the exchange field of the activated neurons in the veins and venules of the brain tissue with a diameter of 60-250 microns [5].

In this work, deliberately simplifying the structure of the components, we considered only the most general principles of the structural construction of ML and the gradient behavior under the influence of ML. Our follow-up works will be focused on the following issues: effects of the methods of external influence, which create and initiate ML formation; the filtration of the input information; the transformation of the character traits of the individual under the ML impact; the priority of ML mainstreaming; tunnelization of thinking and narrowing of consciousness; ML interaction and influence on unconditioned reflex behavior; and objectification of ML markers.

\section{Conclusion}

Regarding MLs as complexes of the psychological, electrical, magnetic, hemodynamic, and temporary equivalents, we define them as properties of a unified psychogenic construct initiating GDB to achieve the ultimate goal: the homeostatic balance of the subject. Localization of the starting point of ST, the timescale for the rate of the result achievement on $\mathrm{ML} / \mathrm{IG}$ that is located outside accessibility, determines the coordinates of the actualization of the structures of GDB, which are unable to achieve the ultimate goal.

We believe that anticipatory, prospective formation of IG, initiating adaptive forms of behavior, is a manifestation of a general principle of "anticipatory response" (the formation of the future in the present moment) inherent for somatic and mental spheres of human ontogenesis. 


\section{References}

1. Jung KG. Archetype and Symbol. M.: Canon; 1991.

2. Berdyaev NA. Slavery and Freedom. M.: ACT; 2006. [in Russian].

3. Toporkov AL. Myth: tradition and psychology of perception. The myth and mythology in modern Russia. Moscow; 2000. [in Russian].

4. Cassirer E. Technique of modern political myths. Vestn Moscow State University. 1990; (Ser. 7, Philosophy) 2:58-65. [in Russian].

5. Kruglov AG, Gebel GY, Vasilyev AY. Intracranial venous pressure as a possible determinant of mental regulation. Int $\mathrm{J}$ Biomed. 2011; 1(4):245-8.

6. Kruglov AG, Gebel GY, Vasilyev AY. Impact of intraextracranial hemodynamics on cerebral ischemia by arterial hypertension (Part 1-2). Int J Biomed 2012; 2(2):89-101.

7. Kruglov A, Vasilyev AYu. Mathematical approaches in studying the ideal image of the goal. Int J Biomed. 2014; 4(1):46-48.

8. Kruglov A, Vasilyev AYu. Psychological image as an active distributed system. J Int Scien Publ Mat Met Tech. 2014; 8:808-11.
9. Shvyrkov VB. Introduction to objective psychology. Neuronal basics of psychics. Moscow: Psychology Institute of RAS (Russian Academy of Sciences); 1995. [in Russian]. 10. Kryzhanovsky GN. Discirculatory pathology of the nervous system. M.: Medinformagenstvo; 2009. [in Russian]. 11. Losev AF. Essays on ancient symbolism and mythology. M.: Nauka; 1993.

12. Rubinshtein SL. Fundamentals of general psychology. St. Petersburg; 2003. [in Russian].

13. Coghill GE. Correlated anatomical and physiological studies of the growth of the nervous system of amphibia. The mechanism of association of Amblystoma punctatum. J Comp Neurol. 1930; 51:311-375.

14. Rajewski KS, Georgiev VP. Mediator amino acids: neurochemical and neuropharmacological aspects. Moscow; 1986. [in Russian].

15. Oleinik VP. Fundamentals of the interaction of physical fields with biological substances. Kharkiv: National Aerospace University; 2006. [in Russian].

16. Lednev VV, Belova NA, Rozhdestvenskaya ZE, Tyros KhP. Biological effects of weak variable magnetic fields and biological precursors of earthquakes. Geophysical Processes and the Biosphere. 2003; 2(1):3-11. [in Russian]. 